\title{
Computational Investigation of Drag Reduction on a Rotationally Oscillating Cylinder
}

\author{
D. Shiels and A. Leonard \\ Graduate Aeronautical Laboratories \\ California Institute of Technology \\ Pasadena, CA 91125, USA
}

\author{
A. Stagg \\ Cray Research, Inc. \\ c/o Jet Propulsion Laboratories \\ MS 301-455, 4800 Oak Grove Drive \\ Pasadenaa, CA 91109, USA
}

\begin{abstract}
The reduction of drag on a circular cylinder in two-dimensional incompressible flow, achieved using oscillatory body rotation, is investigated to reveal the physics responsible for the force reduction. Flow is computationally simulated at Reynolds numbers of 100, 300, and 15000 for a small group of sinusoidal body rotations to gain an understanding of the mechanisms which can control forces in such situations. It is observed that, for certain rotational parameters, drag is reduced over a range of Reynolds numbers from the two-dimensional base flow. At $R e=15000$, an interesting boundary layer instability is observed which could explain the dramatic drag reduction previously noted in an experiment on this flow. Simulations are performed with a high-resolution viscous vortex method which utilizes the particle strength exchange technique for diffusion and multipole expansions for fast summations. Different approaches for computing and analyzing forces will be discussed as well as a 'hybridization' applied to the scheme to allow particle merging in the far wake.
\end{abstract}

\section{Introduction}

Flow over a circular cylinder continues to attract a wealth of attention due to the many complexities that spring from this simple geometry. It is often employed as a benchmark for computational validation against the large existing database such as the flow visualization of Bouard and Coutanceau [1]. Perhaps, though, its greater importance lies in its role as a testbed to study the array of unsteady phenomena associated with bluff body flows. Wake dynamics, unsteady separation points, and shear layer instabilities bedevil attempts to predict these flows, yet the forces in these systems represent a significant concern in practical situations. The circular cylinder provides a 'universal' base problem from which the physics underlying bluff body flows can be explored.

Roshko's [13] measurement of properties of flow over a cylinder triggered an array of experimental investigations aimed at characterizing bluff body flows. This delineation of flow features inspired study of the many avenues of control that bluff body flows suggested. These control studies generally focused on methods to delay separation to influence forces (see the review of Gad-el-Hak and Bushnell [3]) or forcing the flow in some way to control wake structures (see the review of Griffin and Hall [4]). The body of work in this area has identified a variety of successful active and passive control mechanisms, though with an emphasis on global wake structure rather than forces.

A recent experiment by Tokumaru and Dimotakis [14] reveals promise in the previously sparsely investigated (Wu et al. [17]) use of oscillatory body rotation as a drag reducing mechanism (more recently, $\mathrm{Ou}$ [11] considered the effectiveness of rotational oscillation in controlling lift-to-drag 
ratio). Tokumaru and Dimotakis [14] found that a harmonic oscillation could reduce drag on a circular cylinder in $R e=15000$ flow by 80 percent (where $R e=\frac{U_{\infty} D}{\nu}$ ). As with the bulk of past work on bluff body flow control, though, the physical mechanisms behind the observed drag reduction remain in doubt. With experiments limited by an inability to measure fine-scale time dependent boundary layer features and computations also limited by available resources, past studies of bluff body flow control have been forced to rely on global wake observations and time averaged data as a basis for analysis.

Our work attempts to gain a more complete understanding of the physics behind bluff body drag reduction (specifically in the case of rotational oscillation as a control mechanism) by utilizing the detailed data accessible with current computational resources and our highly resolved viscous vortex method. We use instantaneous surface pressure profiles and finely resolved boundary layer velocity fields to unravel the physics of the unsteady boundary layer and near wake. We are able to identify near body behaviors at $R e=100$ and 300 caused by the oscillatory rotation that reduce drag and observe an apparent boundary layer instability at $R e=15000$ that could explain the large drag reduction measured by Tokumaru and Dimotakis [14]. All the flows simulated are strongly forced, and thus one expects two-dimensional effects to dominate the behavior. High resolution simulation at large Reynolds number such as 15000 seems well suited to vortex methods with their ability to accurately resolve the fine-scale features which drive these flows.

\section{Computational method}

We use a Lagrangian viscous vortex method (Koumoutsakos [7], Pepin [12], Winckelmans [16]) with particle merging applied in the wake to facilitate long time computations. Vorticity describes the flow along with a velocity potential field. Vorticity is governed in two-dimensional viscous flow by:

$$
\frac{D \omega}{D t}=\nu \nabla^{2} \omega
$$

along with the requirement that flow velocity on the body surface matches body velocity and flow at infinity matches the free stream.

The velocity is given by the vorticity inversion formula:

$$
\mathbf{u}(\mathbf{x})=\frac{1}{2 \pi} \int \frac{\left(\mathbf{x}^{\prime}-\mathbf{x}\right) \times \omega(\mathbf{x}) \mathbf{e}_{z}}{\left|\mathbf{x}^{\prime}-\mathbf{x}\right|^{2}} d x^{\prime}+\mathbf{U}_{\infty}
$$

The diffusive term is handled by the particle strength exchange technique (Degond and Mas Gallic [2]) where the Laplacian is approximated to second order accuracy in the core size:

$$
\nu \nabla^{2} \omega(\mathbf{x}) \approx \frac{4 \nu}{\pi \sigma^{2}} \int(\omega(\mathbf{x})-\omega(\mathbf{y})) e^{-\frac{|\mathbf{x}-\mathbf{y}|^{2}}{\sigma^{2}}} d \mathbf{y}
$$

The vorticity field is discretized into Gaussian blobs with associated circulations $\Gamma_{i}$ and core size $\sigma$ :

$$
\omega(\mathbf{x})=\sum_{i=1}^{N} \frac{\Gamma_{i}}{\pi \sigma^{2}} e^{-\frac{\left|\mathbf{x}-\mathbf{x}_{i}\right|^{2}}{\sigma^{2}}}
$$

Blobs are convected based on the velocity at their center using second order time marching. Diffusion is resolved using second order time integration and discretized with the approximation 
$\Gamma_{i}=\omega\left(\mathbf{x}_{i}\right) h^{2}$ where $h^{2}$ is the area associated with the blob. An $O(N)$ fast summation method is used to reduce computational costs.

The body boundary condition is satisfied in a physically motivated fashion developed by Koumoutsakos et al. [8] that provides the high resolution desired in this study. It involves solving the above steps on an unbounded domain. A slip velocity results on the body which can be canceled by a vortex sheet $\gamma(s)$ allowed to form on the surface. These two steps are common to many past approaches to the boundary condition in vortex methods. The improvement in the current formulation comes in translating this vortex sheet to the vorticity field accurately. As detailed in Koumoutsakos et al. [8], the vortex sheet is allowed to diffuse into the flow over a time step, thus prescribing an adjustment to the blob circulations to satisfy the slip condition. This is an improvement over the common technique of discretizing the vortex sheet into a layer of blobs on the body surface. A single layer of blobs is not a very accurate discretization of the vorticity field resulting from the sheet diffusing into the flow. It is shown in Leonard et al. [10] that satisfaction of the tangential boundary condition assures adherence to the through-flow condition.

The above formulation leads to difficulties for simulating large wakes as all particles (blobs) are defined with equal size $\sigma$. The wake must therefore be over-resolved to boundary layer scales, leading to prohibitive computational costs. In the $R e=15000$ simulations presented in this paper, 1.7 million particles exist after only 5 radii of linear motion. As will be seen, even at this resolution the boundary layer remains under-resolved. Thus one needs to merge blobs away from the boundary to obtain computational speedup while maintaining resolution near the body. Such an approach can preserve simulation accuracy because relevant scales in the boundary layer will be much smaller than the far wake.

For this study, a simple approach has been implemented to combine particles downstream of the body while keeping a uniform particle size by mapping the existing downstream field to uniform locations. The spacing of the downstream mesh is larger than the original particle spacing, thus rendering a merging technique. The downstream blobs are merged using conservation of circulation and linear impulse as the constraints, Fig. 1. We also treat this downstream region of flow as inviscid because particle strength exchange encounters problems with lack of particle overlap in this technique. The lack of diffusion in the far wake as well as the lesser influence of the far wake on the near body flow permits the use of this hybrid scheme when one's main interest is in forces and not far wake development. More elaborate schemes involving variable particle size will be implemented in future work but initially we wished to consider the simplest approach that guaranteed substantial speedup while maintaining desired accuracy.

This merging scheme was tested by comparing simulations with and without it. In order to choose the position in the wake at which to apply the merging, the level of diffusive interaction in different regions of the flow (indicated by the amount of circulation exchange between particles) was measured in an unmerged $R e=100$ simulation of steady freestream flow (Fig. 2). Based on this result, the merging was begun 12 radii downstream of the cylinder center $(x=12)$ with particles originally spaced by $\Delta x=0.03$ (with $\Delta y=\Delta x$ ) mapped to new locations spaced by $\Delta x=0.05$. From $x=13$ to the edge of the vorticity field, the merging is performed on a mesh with $\Delta x=0.1$. The $x=12-13$ region serves as a transition to keep the field as smooth as possible. A comparison of the vorticity fields from simulation of the flow with and without merging is provided in Figs. 3a-b. One can see that discrepancies in the vorticity field exist in the far wake due to merging and lack of diffusion in the merged case, yet the near body flow appears unaffected. Thus, even at this low viscosity, error does not noticeably propagate upstream. In Figs. 4a-b, it can be seen that the drag and lift forces are virtually unaffected by the merging error. Time steps were executed three times faster in the merged case. Thus the merging scheme used in this study, though somewhat crude, provides a simple modification to increase efficiency without losing accuracy in the flow features of 
interest.

It should also be noted that remeshing is periodically utilized in the region within a few diameters of the body (varying based on Reynolds number) to alleviate the errors caused by strained regions of the Lagrangian mesh. The accuracy of the base scheme has been thoroughly tested in the above references and a more recent paper (Koumoutsakos and Leonard [9]) against theory, experiment and computation and found to provide excellent resolution of wall-bounded viscous flow.

\section{Analysis of forces}

There are two main techniques for computing body forces in an external flow such as that being studied. One (call it method A) obtains force (per unit density) as the time derivative of impulse:

$$
\mathbf{F}=\frac{d}{d t} \int \boldsymbol{\omega} \times \mathbf{x} d \mathbf{x}
$$

Note that this is a global measurement in the sense that all regions of vorticity contribute. Furthermore, errors in vorticity far from the body will be amplified over error near the body due to the spatial multiplier in (5), especially as regards lift. The second approach (call it method B) uses the more localized measurements of pressure and friction on the body:

$$
\mathbf{F}=\oint[(-p \mathbf{n})+(\boldsymbol{\tau} \cdot \mathbf{s})] d s
$$

For vortex methods, one recasts this relation by integrating by parts and using the cylindrical Navier-Stokes equations to yield:

$$
\begin{aligned}
c_{D} & =\frac{2}{R e} \int_{0}^{2 \pi}\left(\frac{\partial \omega}{\partial r}-\omega\right) \sin \theta d \theta \\
c_{L} & =-\frac{2}{R e} \int_{0}^{2 \pi}\left(\frac{\partial \omega}{\partial r}-\omega\right) \cos \theta d \theta
\end{aligned}
$$

These two methods of computation also provide two different frameworks for analyzing forces and their control mechanisms. Method A supports an analysis based on large wake structures and their dynamics well downstream of the body. This has far and away been the preferred approach to date and has supported very informative analysis in some past studies (e.g. Williamson and Roshko [15], Koumoutsakos and Leonard [9]). However, method A is a step removed from the physics causing forces, limiting its ability to fully frame the force evolution. It is simply a global consistency criteria. As was seen in the test of the merging method in Section 2, the dynamics of the downstream flow have limited influence on flow near the body and forces. In fact, the forces plotted in Figs. 4a-b are obtained with method B. Results with method A do not match the plotted curves well due to the global consistency violations caused by the merging and inviscid computation. This also implies a role for using results from both methods of computation as a consistency check for a calculation.

The local approach of method B has the appeal that it springs directly from the physical source of the forces. Control of bluff body forces is really control of the pressure on the body, thus it is certainly preferable to directly observe the quantity one is attempting to influence. To fully describe force control mechanisms, one needs to understand the feedback of the wake on the body pressure, not simply the evolution of the wake. Perhaps method B has been neglected in the past due to the difficulties alluded to in the introduction in obtaining information such as instantaneous pressure distributions. In this current work, we heavily utilize the local perspective of method B to suggest the physical mechanisms driving observed behavior. 


\section{Results}

Due to the time required for high-resolution simulations, only a limited range of flow parameters are used in this study. With a primary motivation of this work being the $R e=15000$ experiment (Tokumaru and Dimotakis [14]), a particular case of that flow is simulated as well as flows with similar parameters at $R e=100$ and 300. Simulations are carried out to their periodic state (where a repeatable shedding pattern is well established) at the lower Reynolds numbers.

Quantities are non-dimensionalized by the freestream velocity and cylinder radius. Thus time represents the number of cylinder radii the body can be considered to have moved linearly during the simulation. The reference frame translates with the body (but not the rotation), so the results can be viewed as either a fixed cylinder held in a flow or a cylinder moving through a steady fluid. Forces are computed using the scheme defined above as method B unless otherwise noted. Pressure distributions on the body surface are obtained by relating the tangential derivative of pressure to the vortex sheet which forms to satisfy the no-slip condition (pressure is non-dimensionalized by $\left.\rho U_{\infty}^{2}\right)$. A reference value of $p(\theta=180)=0.5$ is used to locate the plots. The particle merging discussed above is implemented in the far wake of the $R e=100$ and 300 simulations.

\section{1 $R e=15000$}

As noted above, computations are expensive at this Reynolds number when attempting full resolution. Therefore, only the case with a rotational motion of $\Omega(t)=2 \sin (\pi t)$ was attempted, using 256 processors of the Cray T3D at the Jet Propulsion Laboratory. In their experiment, Tokumaru and Dimotakis [14] found $c_{D} \cong 0.2$ for this controlled flow, which represents a drag reduction of about $80 \%$ from the unforced case. They observed that the forcing led to a more narrow wake (less cross-stream extent). Thus the forcing seemed to delay separation on average, lowering the drag.

We took our simulation to $t=5$ with forcing, and observe a dominant behavior that may underlie the experimentally observed drag reduction. In each half-cycle of forcing, a dipole-like vorticity structure can be observed being ejected from the boundary layer (Figs. 5a-d). The first two structures eventually establish the beginnings of a narrow wake such as observed in the experiment. The pressure distribution at $t=5$ (Fig. 6) on the body exhibits strong recovery on the rear of the cylinder. This suggests that the dipole structures lead to delayed separation, presumably by carrying vorticity out of the boundary layer and thus preventing the establishment of a separated layer.

The resolution for this simulation was $\Delta x \cong 0.0015$ (this was the spacing on the remeshing grid and thus a measure of approximate particle spacing) and $\Delta t=0.004$. The fine scales observed and discrepancy between forces computed with method A and method B indicate that the flow is not fully resolved. Thus, in general, quantitative data such as drag is not provided as its accuracy is in doubt. The same case was simulated with $\Delta x \cong 0.001$ and $\Delta t=0.0025$ out to $t=3.25$. Comparison of the data (e.g. Figs. 7a-b) reveals that in fact the fine scales are under-resolved, leading to some non-trivial differences in the vorticity field, yet the dominant behavior of the dipole structure ejection remains, suggesting that this behavior is accurately captured.

The observed dipole structures are further notable in that they develop at an unusual time, occurring on the side of the cylinder which is moving due to rotation in the same direction as the freestream (positive rotation is counter-clockwise). A close look at the velocity profiles in the boundary layer (Figs. 8a-d) reveals that the body motion turns the boundary layer of the previous half cycle into something of a separated shear layer. This shear region appears to go unstable (observe the $u=0$ contour), thus initiating structures which draw oppositely signed vorticity from the boundary layer into the dipole formation. The velocity field of a vortex dipole explains the 
rapid ejection of the structures from the boundary region.

\section{2 $R e=100$ and 300}

The simulations at lower Reynolds number reached the periodic state of the flow so that timeaveraged quantities could be obtained. Below is a listing of the cases simulated and the resulting time-averaged drag coefficient. The cases were chosen to compare the results of the forcing to the $R e=15000$ case. The simulations without rotational forcing were started with a brief rotational oscillation to induce the asymmetry necessary for vortex shedding.

\begin{tabular}{lc}
\hline \hline \multicolumn{1}{c}{ Parameters } & Average Drag Coefficient \\
\hline$R e=100, \Omega=0$ & 1.33 \\
$R e=100, \Omega=0.5 \sin (\pi t)$ & 1.33 \\
$R e=100, \Omega=2 \sin (\pi t)$ & 1.28 \\
$R e=100, \Omega=4 \sin (\pi t)$ & 1.18 \\
$R e=100, \Omega=2 \sin (\pi t / 3)$ & 1.35 \\
$R e=300, \Omega=0$ & 1.38 \\
$R e=300, \Omega=2 \sin (\pi t)$ & 1.13 \\
\hline \hline
\end{tabular}

The trend in this data is for reduced drag due to rotational control, but only for larger amplitudes and frequencies of oscillation. In Figs. 9a-f, the end states of these flows are provided, revealing very little difference in the far wake structures (with one exception noted below) despite the differences in drag. It should be noted that the shedding frequencies in the unforced cases, $S_{f}=\frac{f D}{U_{\infty}}$, accurately matched experimental data $\left(S_{f}=0.165\right.$ for $R e=100$ (cf. Hammache and Gharib [5]), $S_{f}=0.204$ for $R e=300)$.

The drag coefficient for $R e=100$ matches experimental results. The $R e=300$ case is in the three-dimensional regime, but the drag does match other two-dimensional computational results (Henderson [6]).

Using the global approach for force analysis (method A), the strength of the wake structures must be measured to explain the drag behavior as there is virtually no difference in eddy locations for most cases. In fact, the one flow with a significantly different wake (Fig. 9d) actually has a difference in $c_{D}$ of only 0.02 from the base case. Measurement of the circulation of a wake structure for $R e=100, \Omega=4 \sin \pi t$, did reveal net circulations in the wake eddies $17 \%$ less than the base case. It is not clear, however, how to prove a link between the control mechanism and noted reduced wake circulation. Nor is it clear why reduced wake circulation would effect the forces on the body beyond the fact that Eq. 5 dictates it.

Consider instead analysis of the drag reduction from the local perspective (method B). The time-averaged effects of the rotational control can be observed in profiles of time-averaged pressure on the body (Figs. 10a-d). Increasing the rotational amplitude yields greater pressure recovery and thus lower drag. One expects the Koanda effect to trigger recovery on one half of the cylinder (where the body rotates in the direction of the freestream, thus delaying separation), but adverse effects on the drag are likewise anticipated on the other half.

To resolve the situation, consider the instantaneous vorticity, pressure, and streamline fields for the base $R e=100$ flow $(\Omega=0)$ and the flow at $R e=100$ with the largest drag reduction $(\Omega=4 \sin \pi t)$ (Figs. 11a-f). At this time $(t=68.6)$, the cylinder is rotating counterclockwise. Note the expected pressure recovery from the Koanda effect on the lower $(y>0)$ side, but also a noticeable recovery on the opposite side (pressure recovery is used here to refer to the increase in pressure on the downstream face from the lowest pressure position, not to an increase in the 
value of the base pressure). This unexpected recovery explains the measured drag reduction since an early separation and dead-water region does not form instead. Although the base pressure is not significantly different between Figs. 11e and 11f, there is more suction on the upstream face in Fig. 11f due to the lower minimum pressure, which combines with the stronger pressure recovery in that case to yield a lower drag. Of course, this effect works in concert with the higher base pressure observed in Fig. 10 in the reduced drag flows. But the key to the base pressure behavior is this pressure recovery on the downstream face that induces the higher base pressures.

The streamlines and vorticity field seem to indicate the cause of this surprising behavior. The oscillation creates a layer of vorticity in one half-cycle of forcing, which, for large enough amplitude, effectively cuts off the separated layer feeding the wake. Thus, note in the streamlines that flow outside the boundary layer on both sides of the cylinder remains attached longer than in the base case. On one side, this delay in separation is due to the Koanda effect, on the other it seems to be caused by the interruption of the separated shear layer. For $R e=300$, the same behavior is observed, though the lesser viscosity seems to aid in 'pinching off' the separated layer, thus leading to larger drag reduction than at $R e=100$ (Figs. 12a-b).

\subsection{Efficiency of drag reduction}

Although this study was aimed at revealing the physics behind drag reduction, the efficiency (power savings) of the system is also measured. Define a power saving ratio $P_{S R}$ as:

$$
P_{S R}=\frac{\text { Power saved through drag reduction }}{\text { Power spent on rotational motion }}
$$

For this geometry, assuming a cylinder of negligable mass, the ratio is given by:

$$
P_{S R}=R e \frac{\Delta c_{D}}{\left\langle-\dot{\theta}\left(-4 \dot{\theta}+\int_{0}^{2 \pi} \omega d \theta\right)\right\rangle}
$$

where:

$$
\begin{aligned}
\Delta c_{D} & =\text { reduction in drag coefficient } \\
\dot{\theta} & =\text { angular velocity of body } \\
\langle\rangle & =\text { average over a shedding cycle }
\end{aligned}
$$

For the $R e=300$ case $\Omega=2 \sin \pi t, P_{S R}=0.29$ (using the reduction over the $2 \mathrm{D}$ base flow). Thus at lower Reynolds numbers, this method of drag reduction is not suited for practical implementation (of course, the real 3D drag at $R e=300$ makes it even less efficient). Note, though, that the following scaling holds if $\Delta c_{D}$ is considered a constant:

$$
\delta \propto \frac{1}{\sqrt{R e}} \quad \omega \propto \sqrt{R e} \quad P_{S R} \propto \sqrt{R e}
$$

where $\delta=$ boundary layer thickness. Further, trends suggest the drag savings will increase with Reynolds number, so that at higher Reynolds numbers this may be an efficient system.

\section{Conclusions}

Rotational oscillation with appropriate parameters appears to be an effective drag reducing agent for cylinder flow over a range of Reynolds numbers, not just near the critical regime (i.e. transition 
to turbulence). A hybrid scheme for efficient handling of the far wake has allowed for long-time, well-resolved computations with a vortex method. At Reynolds number 15000 , an interesting instability caused by rapidly oscillating body motion purges the boundary layer of vorticity. This mechanism explains the delayed separation and large drag reduction experimentally observed for the flow. It also could explain the dependencies on the frequency and amplitude of the rotational oscillation found in the experiment. At lower Reynolds numbers (100 and 300), this instability is not observed, but drag reduction still results from control with amplitudes and frequencies that efficiently cut off separating layers, allowing some pressure recovery to complement the Koanda effect on the downstream face. The identification of both of these drag reducing mechanisms relied on an attempt to reveal the physics underlying measured force behavior, and exemplify the importance of small scale effects embedded in near-body regions. The enhanced understanding of the mechanisms behind the drag behavior reveals that they could also be applied in bluff body flows in general and are not techniques specialized to the cylinder.

\section{Acknowledgements}

One of us (D.S.) would like to thank Dr. Petros Koumoutsakos for much help and many valuable discussions prior to this project. This work was supported through the Office of Naval Research through Grant No. N00014-94-1-0793. Computer time was provided by the Jet Propulsion Laboratory Supercomputing Project (funded from the NASA Offices of Mission to Planet Earth, Aeronautics, and Space Sciences) . One of us (D.S.) was an NDSEG fellow (DoD Grant \#DAAH0493-G-0281). A parallel version of the vortex code was developed for the Cray T3D through Cray Research's Parallel Applications Technology Program.

\section{References}

[1] Bouard, R. and Coutanceau, M., "The Early Stage of Development of the Wake behind an Impusively Started Cylinder for $40<R e<104, "$ J. Fluid Mech., 101, pp. 583-607, 1980.

[2] Degond, P. and Mas-Gallic, S., "The Weighted Particle Method for Convection-Diffusion Equations," Math. Comp., 53, pp. 485-526, 1989.

[3] Gad-el-Hak, M. and Bushnell, D., "Separation Control: Review," J. Fluids Eng., 113, pp. 5-30, 1991.

[4] Griffin, O. and Hall, M., "Review-Vortex Shedding Lock-on and Flow Control in Bluff Body Wakes," Trans. ASME, 113, pp. 526-537, 1991.

[5] Hammache, M. and Gharib, M., "A Novel Method to Promote Parallel Vortex Shedding in the Wake of Circular Cylinders," Phys. Fluids A, 1, pp. 1611-1614, 1989.

[6] Henderson, R., "Details of the Drag Curve near the Onset of Vortex Shedding," Phys. Fluids, 7, pp. 2102-2104, 1995.

[7] Koumoutsakos, P., Direct Numerical Simulations of Unsteady Separated Flows using Vortex Methods, Ph.D. thesis, Caltech, 1993.

[8] Koumoutsakos, P., Leonard, A. and Pepin, F., "Boundary Conditions for Viscous Vortex Methods," J. Comp. Phys., 113, p. 52, 1994. 
[9] Koumoutsakos, P. and Leonard, A., "High-Resolution Simulations of the Flow around an Impulsively Started Cylinder using Vortex Methods," J. Fluid Mech., 296, pp. 1-38, 1995.

[10] Leonard, A., Koumoutsakos, P. and Winckelmans, G., "Vortex Methods for Three-Dimensional Separated Flows," Proc. 14th Int'l. Conf. Num. Meth. Fluid Dynamics, Bangalore, India, Springer-Verlag, Lecture Notes in Physics, 1994.

[11] Ou, Y., "Control of Oscillatory Forces on a Circular Cylinder by Rotation," NASA CR-187619, 1991.

[12] Pepin, F., Simulation of the Flow past an Impulsively Started Cylinder using a Discrete Method, Ph.D. thesis, Caltech, 1990.

[13] Roshko, A., "On the Drag and Shedding Frequency of Two-Dimensional Bluff Bodies," NACA TN 3169, 1954.

[14] Tokumaru, P. and Dimotakis, P., "Rotary Oscillation Control of a Cylinder Wake," J. Fluid Mech., 224, pp. 77-90, 1991.

[15] Williamson, C. and Roshko, A., "Vortex Formulation in the Wake of an Oscillating Cylinder," J. Fluids Struct., 2, pp. 355-381, 1988.

[16] Winckelmans, G., Topics in Vortex Methods for the Computation of Three- and Two-Dimensional Incompressible Unsteady Flows, Ph.D. thesis, Caltech, 1989.

[17] Wu, J., Mo, J. and Vakili, A., "On the Wake of a Cylinder with Rotational Oscillations," AIAA Rep. 89-1024, 1989. 


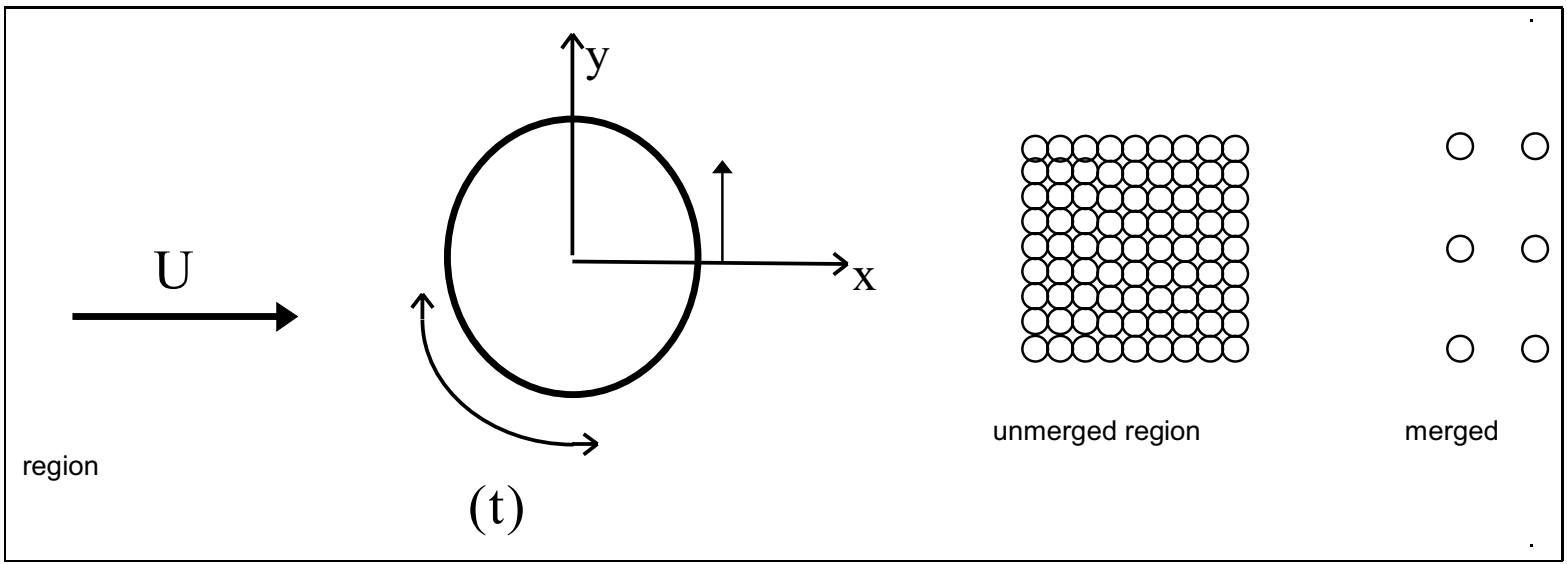

Figure 1: Configuration of system. Particle merging is applied well downstream of the body.

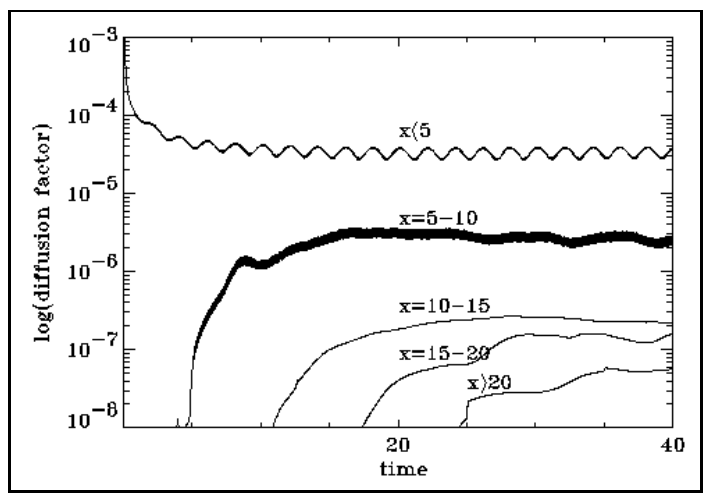

Figure 2: The average circulation exchange between particles in different regions of the flow (used as a measure of the significance of diffusion in different regions of the flow).

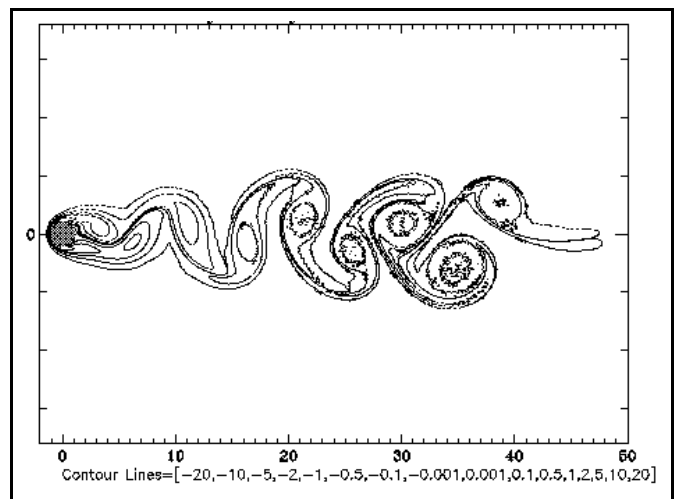

Figure 3a: Vorticity field with particle merging applied and diffusion neglected for $x>12(t=50)$.

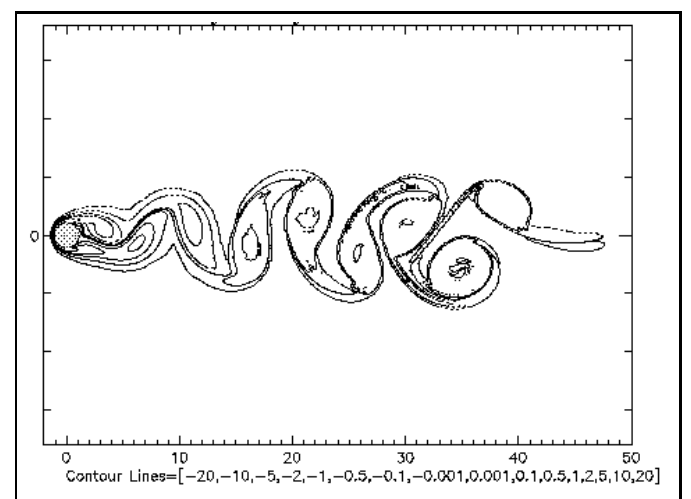

Figure 3b: Vorticity field without particle merging and diffusion applied everywhere $(t=50)$. 


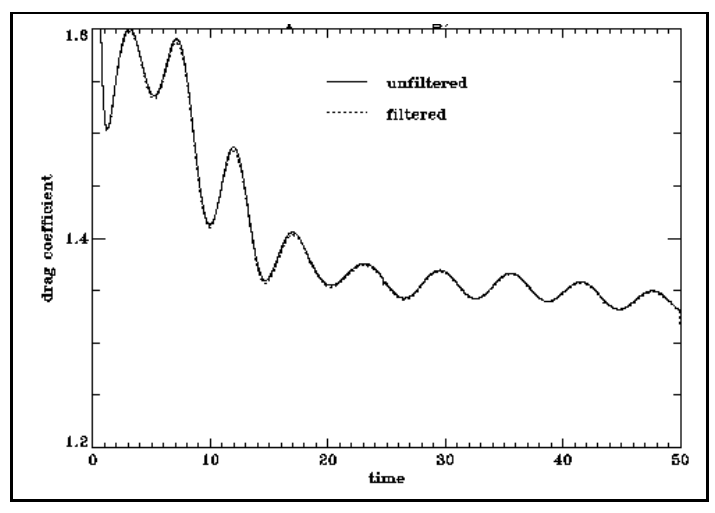

Figure 4a: Comparison of drag between simulations for which vorticity fields are plotted in Figs. 3a-b (filtered is used here to mean merged).

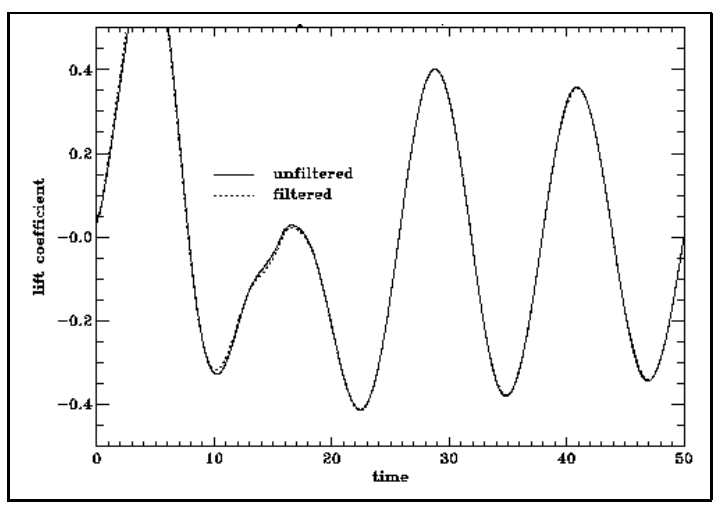

Figure 4b: Comparison of lift for simulations used in Fig 4a.
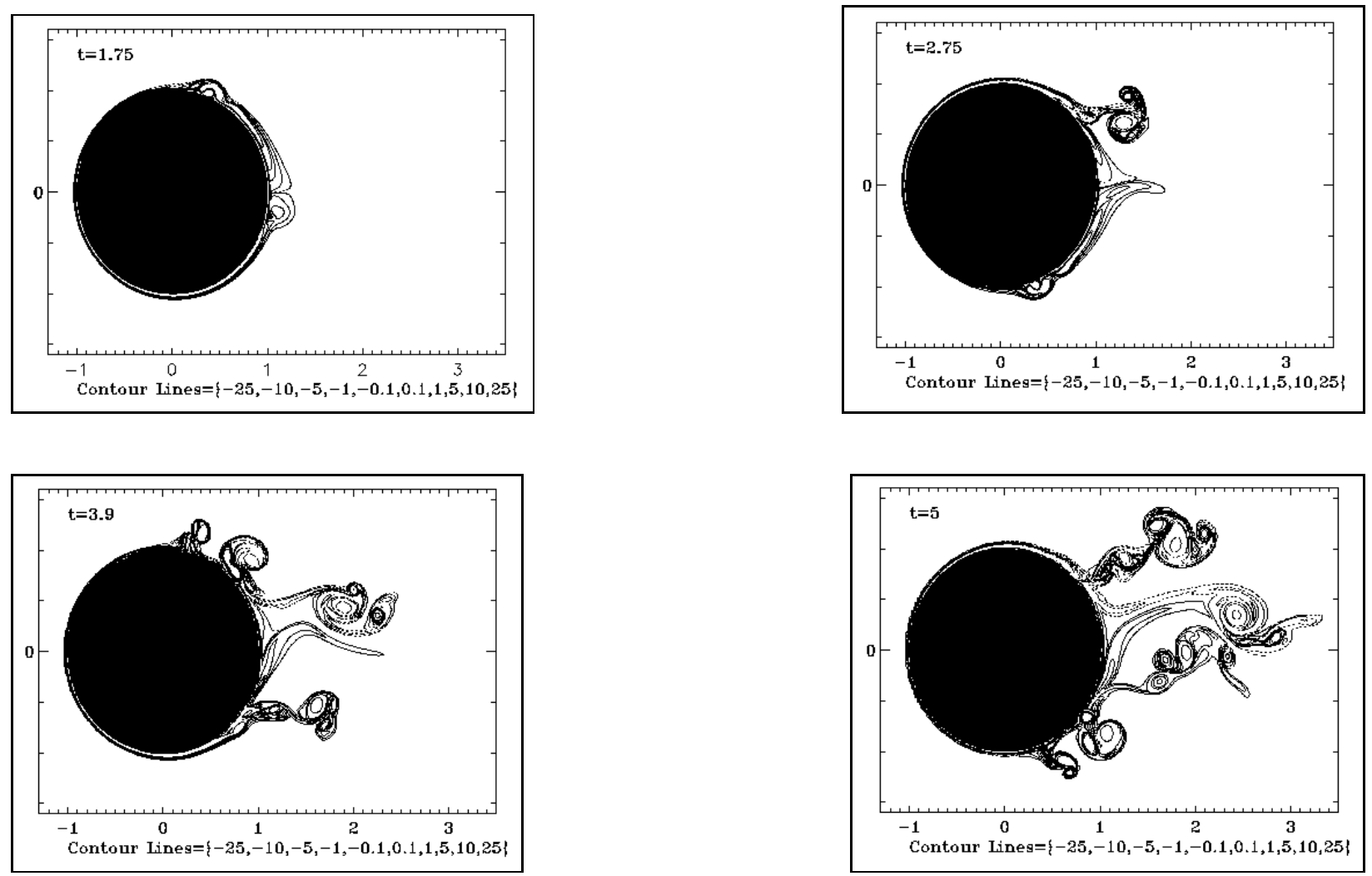

Figure 5: (a-d) Vorticity field for $R e=15000$ simulation. $(\Omega=2 \sin \pi t)$. 


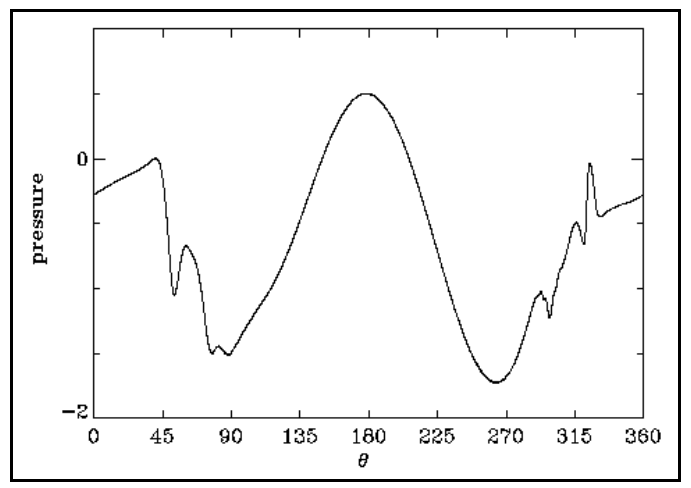

Figure 6: Pressure on the body surface at $t=5, R e=15000$.

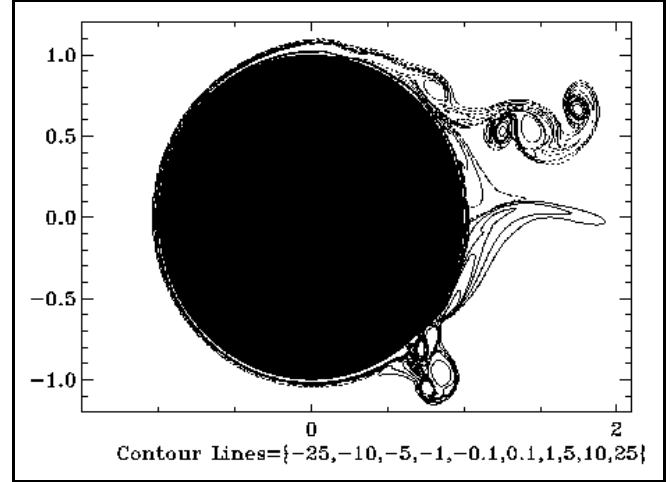

Figure 7a: $R e=15000, \Delta x \cong 0.0015$, $\Delta t=0.004, t=3.15$.

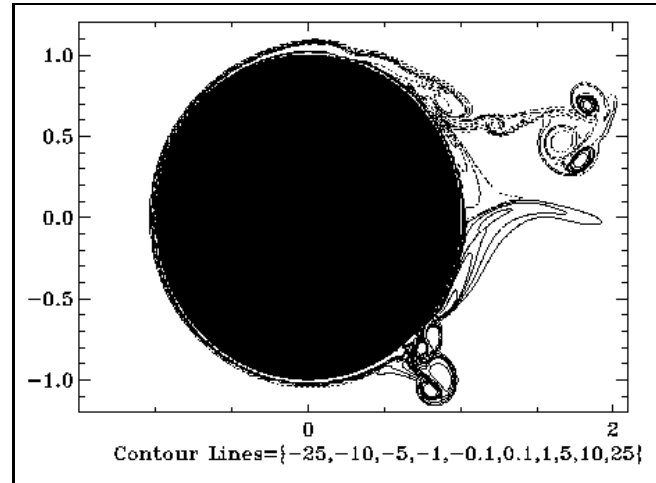

Figure 7b: $R e=15000, \Delta x \cong 0.001$, $\Delta t=0.0025, t=3.15$. 

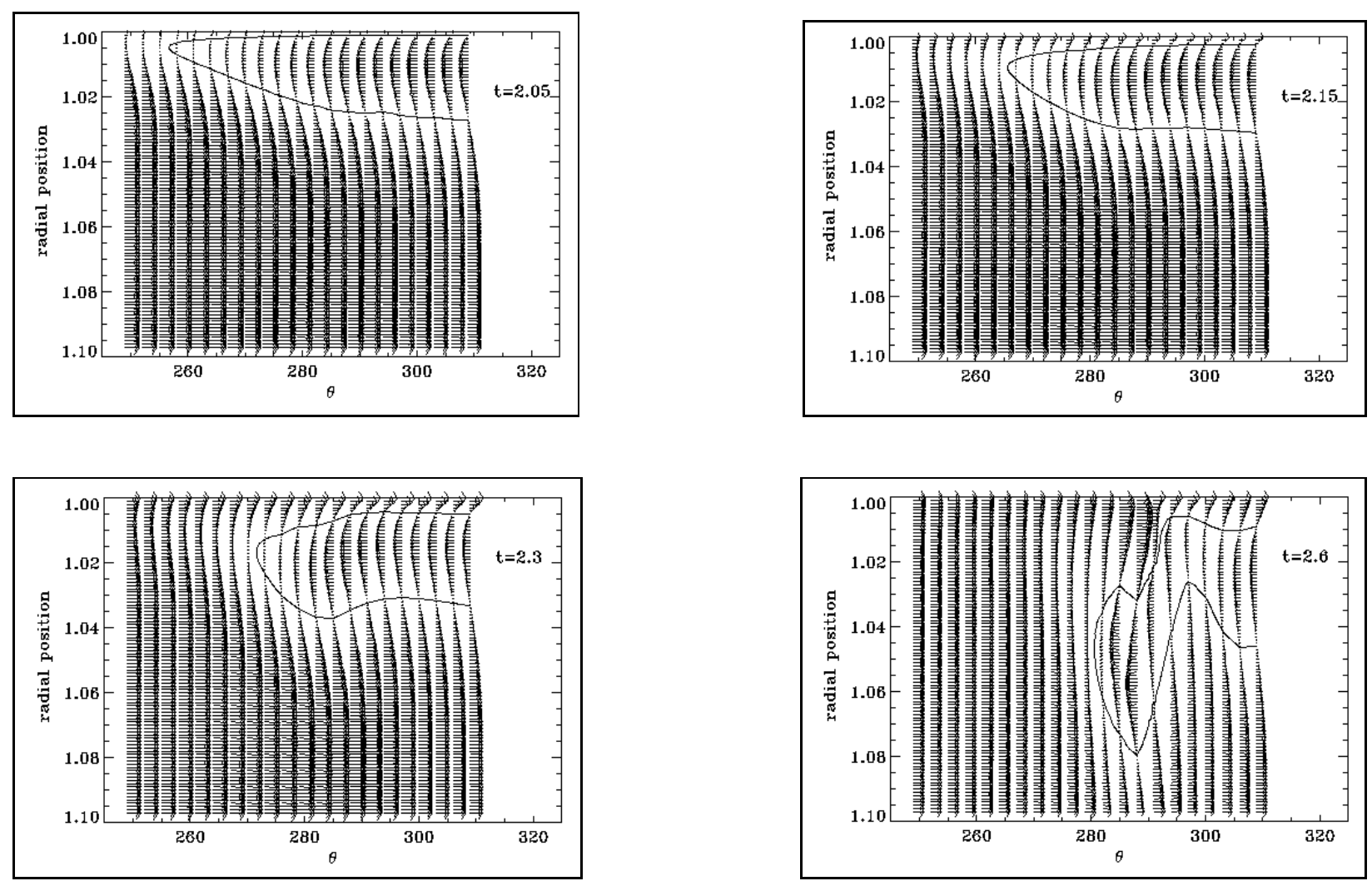

Figure 8: (a-d) Velocity profiles in the boundary region on the 'lower' half $(y<0)$ of the cylinder along with the $u=0$ contour. Velocity vectors are to scale relative to each other $(r=1$ corresponds to the surface). 

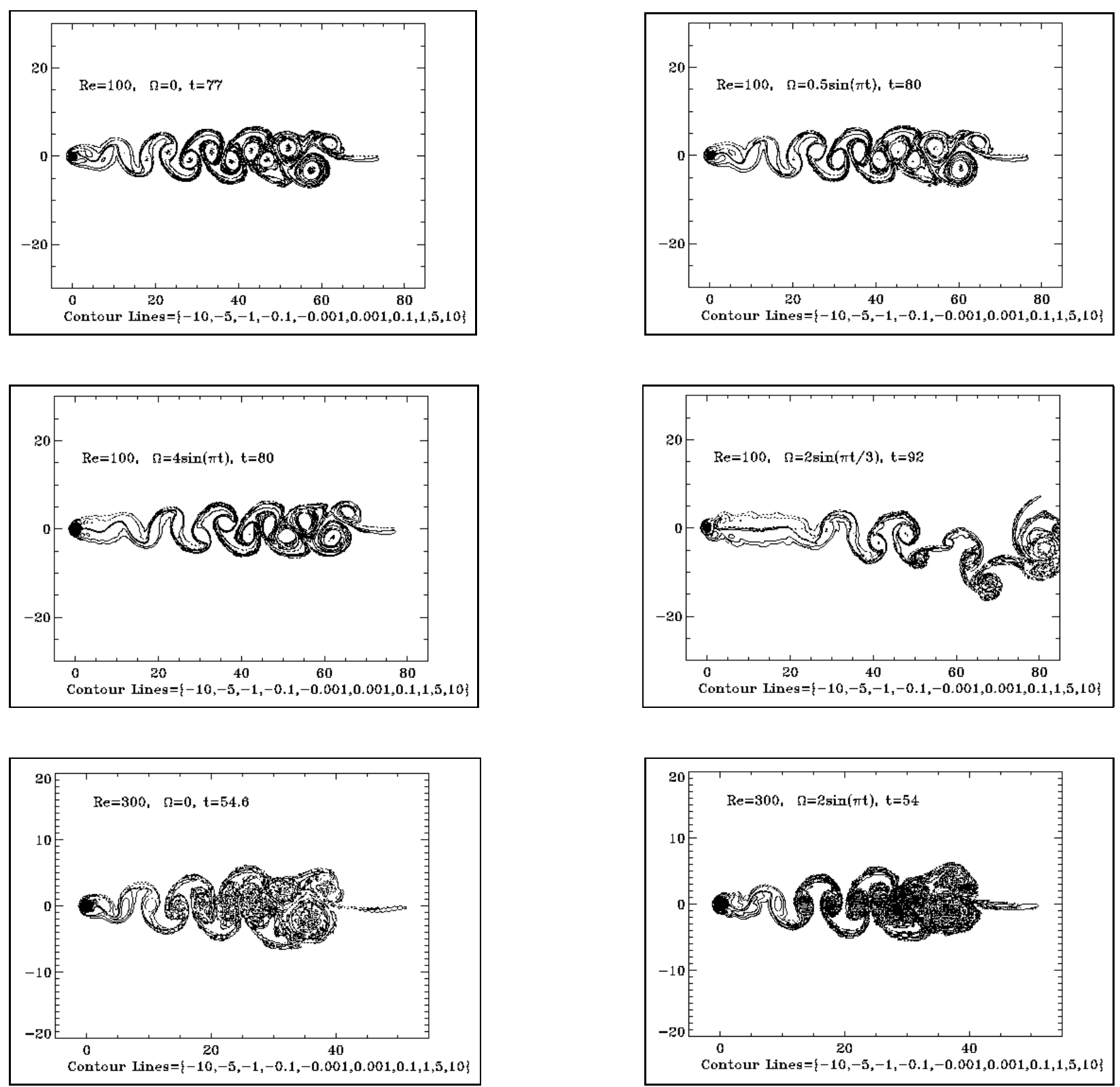

Figure 9: (a-f) Final computed vorticity fields for different cases. 

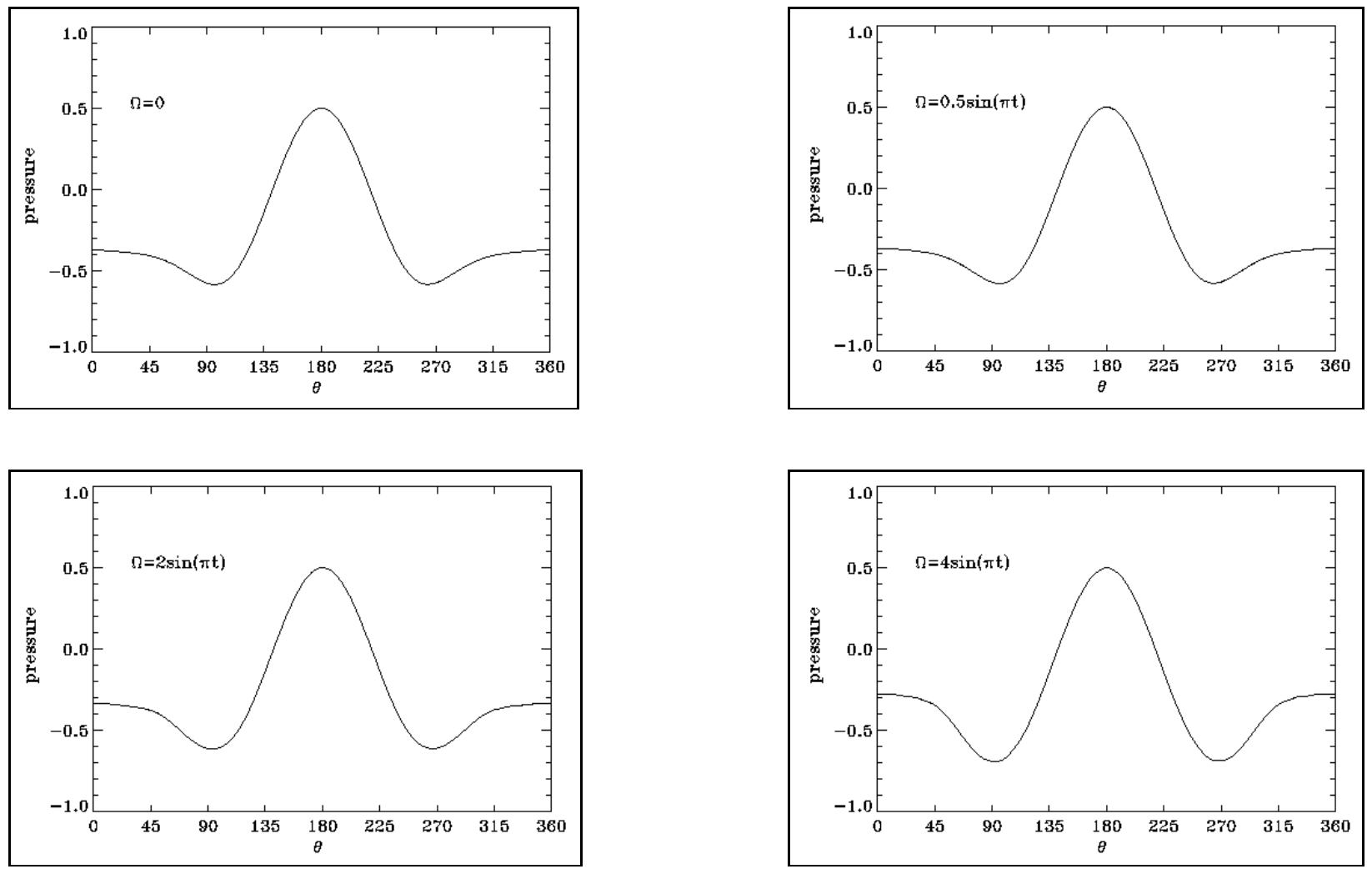

Figure 10: (a-d) Body pressure distributions averaged over one shedding cycle. $R e=100$. 



Figure 11: (a-f) Vorticity, streamlines, and body pressure at $t=68.6$. 

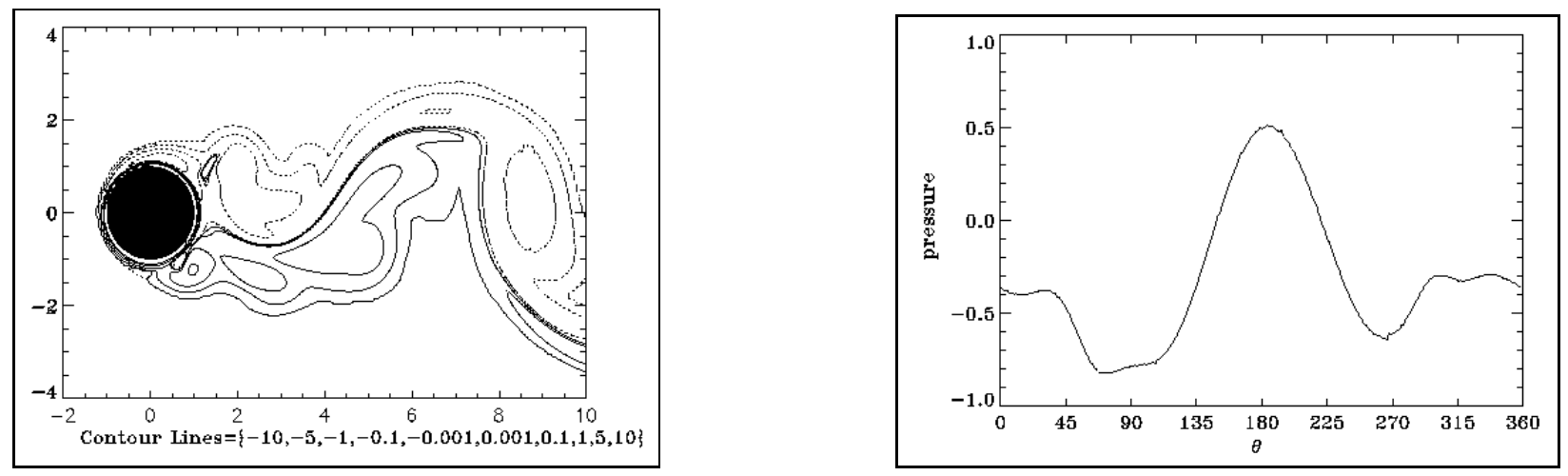

Figure 12: (a-b) Vorticity at $t=63.3$ for $R e=300$. 